The Cardiology 9 (1): 1-6, 2014

ISSN: 1811-8194

(C) Medwell Journals, 2014

\title{
Dental Tissue-Derived Stem Cells Exerts Therapeutic Effects on Chronic Myocardial Infarction Model of Rabbit
}

\author{
${ }^{1}$ Soontaree Petchdee, ${ }^{2}$ Nakrob Pattanapon, ${ }^{2}$ Ratikorn Bootcha, \\ ${ }^{3}$ Petcharin Srivattanakul and ${ }^{4}$ Taweesak Songserm \\ ${ }^{1}$ Department of Large Animal and Wildlife Clinical Sciences, \\ Faculty of Veterinary Medicine, Kasetsart University, \\ 73140 Kamphaeng Saen Campus, Bangkok, Thailand \\ ${ }^{2}$ Kasetsart University Veterinary Teaching Animal Hospital, Kamphaeng Saen, Thailand \\ ${ }^{3}$ BioEden Asia Tooth Cell Bank, Bangkok, Thailand \\ ${ }^{4}$ Department of Pathology, Faculty of Veterinary Medicine, Kasetsart University, \\ Bang Khen, Bangkok, Thailand
}

\begin{abstract}
Coronary artery disease is a common precursor to sudden cardiac death worldwide. Advanced symptoms usually include Myocardial Infarction (MI) due to atherosclerosis of coronary arteries. To repair or regenerate lost myocardium and coronary vasculature, stem cell transplantation is a promising therapeutic approach for the treatment of coronary heart diseases. In this study, the therapeutic effects of multipotent Stem Cells from Human Exfoliated Deciduous teeth (SHED) were examined. The 30 adult male New Zealand White rabbits underwent a left thoracotomy approach for producing chronic infarcted heart. The marginal branch of the left circumflex coronary artery was ligated over 8 weeks to produce an ischemic area of $20-25 \%$ of the Left Ventricle (LV). SHEDs were freshly prepared and $1.0 \mathrm{~mL}$ of $10^{6}$ cells were injected to each of eight rabbits via the marginal ear vein. Echocardiography and Heart Rate Variability (HRV) were measured to reflect cardiac function. The infarcted size measurements were performed at the end of each experiment. The SHED treatment groups show significant improvement in cardiac autonomic tone and reduction in infarcted size. Dental tissue derived stem cell transplantation confirmed a restoration of the heart. The results suggest that SHED could provide an alternative selection of the precursor cells for cardiac repair.
\end{abstract}

Key words: Mesenchymal stem cells, myocardial infarction, cardiac repair, therapeutic, dental tissue

\section{INTRODUCTION}

Heart disease is the world's leading cause of death threatening more human lives than any other disease (Roger et al., 2012). Advanced symptoms usually include Myocardial Infarction (MI) due to atherosclerosis of coronary arteries. Even after successful coronary revascularization cell death continues and the loss of cardiomyocytes ultimately leads to progressive ventricular dilation and heart failure. Stem cell transplantation becomes widely studied for therapeutic approaches in the field of regenerative medicine and also for repairing damaged myocardium (Gepstein, 2002). Mesenchymal Stem Cells (MSCs) transplanted into myocardial scar improve the heart functions and prevent the further episodes of myocardial infarction (Brehm et al., 2002; Caspi and Gepstein, 2006; Tomita et al., 1999). However, it is still unclear whether effects are the result of differentiation of stem cells into cardiomyocytes or myocardial preservation (Fuchs et al., 2006). MSCs can be isolated from a variety of organs and tissues such as bone marrow, brain, skin, hair follicle, skeletal muscle and dental pulp (Da Silva Meirelles and Nardi, 2003).

However, the clinical use of several stem cells has been controversial and limited due to the ethical concerns (Petersen and Niklason, 2007). Recently, dental tissue derived stem cells such as Dental Pulp Stem Cells (DPSCs) and Stem cells from Human Exfoliated Deciduous teeth (SHED) have been suggested as a novel alternative resource for cell therapies and tissue engineering (Huang et al., 2009). These dental tissue derived stem cells have Mesenchymal Stem Cell (MSC) qualities including the capacity for self-renewal and multilineage differentiation potential. Dental MSC like stem cells are not only derived from a very approachable tissue resource but are also able to supply enough cells for clinical application (Gandia et al., 2008). The objective of this study was to determine whether SHED could be used for myocardial regeneration in a rabbit model of chronic myocardial infarction.

Corresponding Author: Soontaree Petchdee, Department of Large Animal and Wildlife Clinical Sciences, Faculty of Veterinary Medicine, Kasetsart University, 73140 Kamphaeng Saen Campus, Bangkok, Thailand 


\section{MATERIALS AND METHODS}

Animals: The study was conducted in thirty New Zealand white rabbits and approved by the Ethical Committee for Animal Experiments, Kasetsart University, Thailand. Clinical evaluation consisted of physical examination, electrocardiography and complete blood cell count. Animals were anesthesized with isoflurane ( $5 \%$ induction, $2 \%$ maintenance), intubated and connected to a ventilator. Ventilation was done with a tidal volume of $50 \mathrm{~mL}$ at frequency $36 \mathrm{bpm}$. Left lateral thoracotomy was performed along the fourth intercostal space and the pericardium was cut at the level of and parallel with the atrium. There are two anatomical patterns of rabbit coronary artery branching pattern; bifurcation and trifurcation as illustrated in Fig. 1. The marginal branch of the Left Circumflex Coronary Artery (LCA) was ligated to produce $20-25 \%$ of infarction and the thoracotomy was closed subsequently after $20 \mathrm{~min}$ of LCA ligation. Animals were maintained for 8 weeks for establishment as Chronic Myocardial Infarction Models.

Measurement of heart rate variability: Electrocardiogram recording was measured in 30 rabbits throughout the experiment. Electrocardiograms (ECGs) were measured and recorded before and after SHEDs administration by iWorx 214. Animals were anesthetized with $2 \%$ isofurane in $70 \%$ $\mathrm{N}_{2} \mathrm{O}$ and $30 \% \mathrm{O}_{2}$ by a face mask. ECGs data were collected for a continuous 15-20 min interval for each rabbit without physical movements and positional changes. Heart Rate Variability (HRV) was calculated using a Fast Fourier Transform (FFT) based Non-Parametric algorithm.

Cardiac function measurement: Echocardiography was performed before and 8 weeks after surgery for animals subjected to chronic myocardial infarction using $10 \mathrm{MHz}$ transducer of GE Vivid s5 ultrasonography

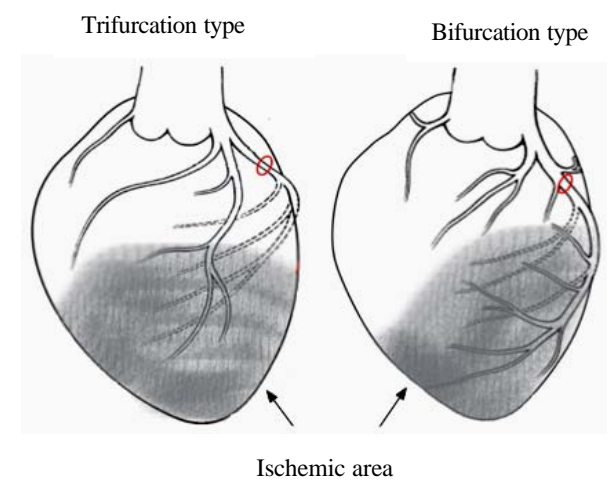

Fig. 1: Anatomical branching patterns of rabbit coronary artery: bifurcation or trifurcation system (General Electric Company; USA). The cardiac function were evaluated from parameters such as: aortic root size, Left Ventricular End Diastolic and Systolic Diameter (LVEDd and LVESd), Interventricular Septum Thickness at Diastolic and systolic (IVSTd and IVSTs), Left Ventricular Diastolic and systolic diameter (LVDd and LVDs), Left Ventricular Wall thickness at Diastolic and Systolic (LVPWd and LVPWs) and Fractional Shortening $(\mathrm{FS} \%)$.

Cells transplantation: Stem cells from human exfoliated deciduous teeth (SHED) were supported from BioEden Asia Tooth Cell Bank. Cells were cultured in Dulbecco's modified Eagle's medium (Sigma-Aldrich, St. Louis, MO) supplemented with $10 \%$ fetal bovine serum (Invitrogen) and $1 \%$ penicillin/streptomycin at $37^{\circ} \mathrm{C}, 5 \% \mathrm{CO}_{2}$. Cells were harvested and collected from the culture at $80 \%$ confluency via trypsin-EDTA treatment. SHED at passages 4-8 were characterized by fluorescence activated cell sorting analysis. All transplantation techniques were performed under aseptic conditions. Approximately, $1.0 \times 10^{6}$ (SHED) cells were administered intravenously through rabbit marginal ear vein at 4 weeks after LCA ligation for a single transplantation and at 2 and 4 weeks after LCA ligation for multiple transplantations.

Measurement of infarct size and histology: Animals were subsequently euthanized and the hearts were immediately removed and sectioned into $2 \mathrm{~mm}$ thick short-axis slices from the apex towards the base of the heart as illustrated in Fig. 2. Myocardial sections were submerged and incubated in Triphenyl Tetrazolium Chroride solution (TTC) for $10 \mathrm{~min}$ at $37^{\circ} \mathrm{C}$. Neutral formalin fixation was performed for pathological analysis. Cardiac tissues were paraffin wax embedded and sectioned into slices which were stained with Hematoxylin and Eosin (H\&E). Infarct scar area $(\%)$ was calculated from the ratio of the area of infarct and total area of LV myocardium.
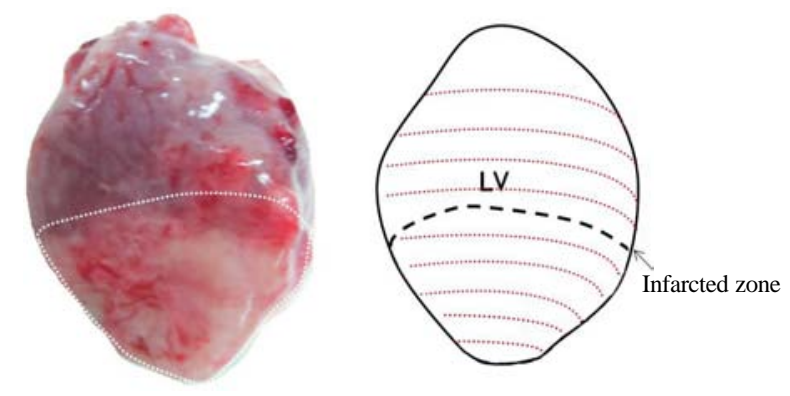

Fig. 2: Left Ventricle (LV) was sliced into $2 \mathrm{~mm}$ thick from apex to base of the heart, dash line represents boundary between normal and infarcted myocardium 
Statistical analysis: Animals were randomly divided in four experimental groups, consist of group 1 (control), rabbits given normal saline alone without stem cell administration $(\mathrm{n}=8)$, group 2 (SHED-single), rabbits given SHEDs $\left(1 \times 10^{6}\right)$ injected intravenously at 4 weeks after coronary artery occlusion $(\mathrm{n}=8)$, group 3 (SHED-multiple), rabbits given SHEDs $\left(1 \times 10^{6}\right)$ injected intravenously at 2 and 4 weeks after coronary artery occlusion $(\mathrm{n}=8)$ and group 4 Sham-operated rabbits performed thoracotomy without coronary occlusion $(n=6)$. Group data were analyzed using a one way ANOVA test where $\mathrm{p}<0.05$ was considered significant. Data in this study are presented using mean \pm Standard Error of the Mean (SEM).

\section{RESULTS AND DISCUSSION}

Characterizations of mesenchymal stem cells: The positive expression of surface markers was $100 \%$ for Strol (Fig. 3). The Mesenchymal Stem Cells (MSCs) adhered to plastic culture dishes and formed fibroblast-like colonies as shown in Fig. 3a.

Effect of SHEDs given 2 and 4 weeks after ischemia Infarcted size and neovascularization: The comparison of infarcted size showed better recovery of scar tissue (\%) between single and multiple doses of SHEDtransplantations: $10.9 \pm 0.02$ vs. $9.7 \pm 0.02$ and showed significant reduction in the scar tissue $(\%)$ between
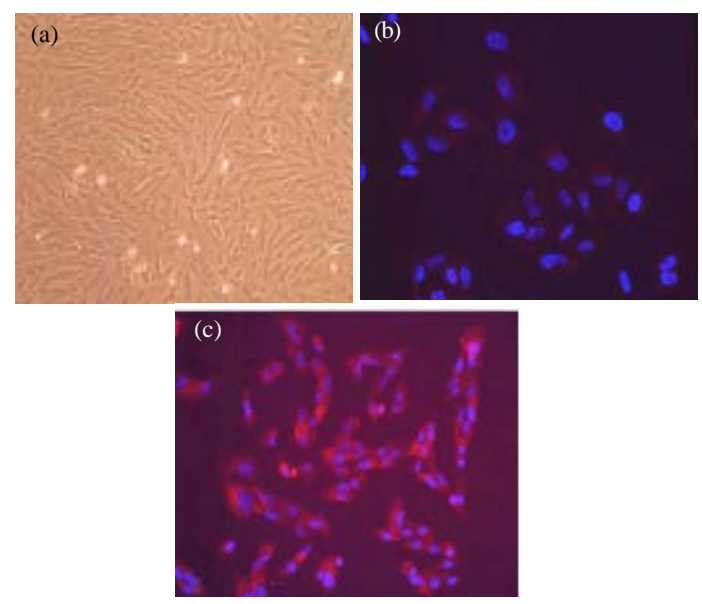

Fig. 3: a) Healthy SHEDs have stem-cell characteristics. b) Fluorescene-activated cell sorting analysis showed that SHEDs contained 100\% STRO-1 positive cells; c) when compared with Hela cervical carcinoma cells in. Blue is Dapi stained nuclei while red is Stro-1 cell surface marker by Cy3 labelled antibody control groups and SHED-transplanted groups (single and multiple injections ): $19.9 \pm 0.03$ vs. $10.9 \pm 0.02 ; \mathrm{p}<0.05$ and $19.9 \pm 0.03$ vs. $9.7 \pm 0.02 ; \mathrm{p}<0.01$, respectively. The infarcted size measurements in Fig. 4 are expressed as mean \pm SEM and represented the mean values for 8 animals. Hematoxylin-Eosin (H\&E) staining showed myocardial cell loss in the infarct area in control heart section (Fig. 5b). An increased number of myocardial cells and capillaries based on H\&E staining were presented in SHED-transplanted group as shown in the representative sections (Fig. $5 \mathrm{c}$ and $\mathrm{d}$ ).

Cardiac function and autonomic modulation: At 2 and 4 weeks after SHED transplantation the heart rate variability and Fraction Shortening (FS\%) in control, SHED-single and SHED-multiple transplantation groups are shown in Fig. 6a-c, respectively. As shown in Fig. 6a, heart rate measurements showed significant difference among the four groups. There were no significant difference in heart rate variable echocardiography and electrocardiography parameters as well as in the blood profiles were seen among the four groups, although, SHED transplanted group tended to improve the heart rate and autonomic nervous system balance.

Previous studies have reported an improvement in cardiac function after acute myocardial infarction by intracardiac transplantation of Dental Pulp Stem Cells (DPSC) and multiple intravenous injection of Human Cord Blood Cell (HCBC) (Gandia et al., 2008; Henning et al., 2007). In this study, researchers validated the efficacy and safety of SHEDs transplantation after myocardial infarction in a rabbit model of chronic myocardial infarction.

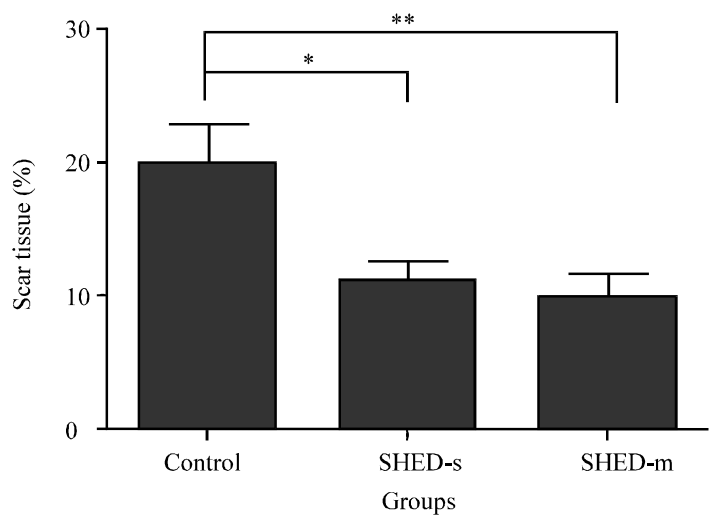

Fig. 4: Effects of SHEDs transplantation on infarcted size; It represents heart section from 8 weeks infarcted SHED-untreated (Control) compared to single SHED-treated (SHED-s) and multiple SHED-treated (SHED-m). Values are mean \pm SEM corresponding to $\mathrm{n}=8$ for control and SHEDs transplanted groups. ${ }^{*} \mathrm{p}<0.05$ and ${ }^{* *} \mathrm{p}<0.01$ 

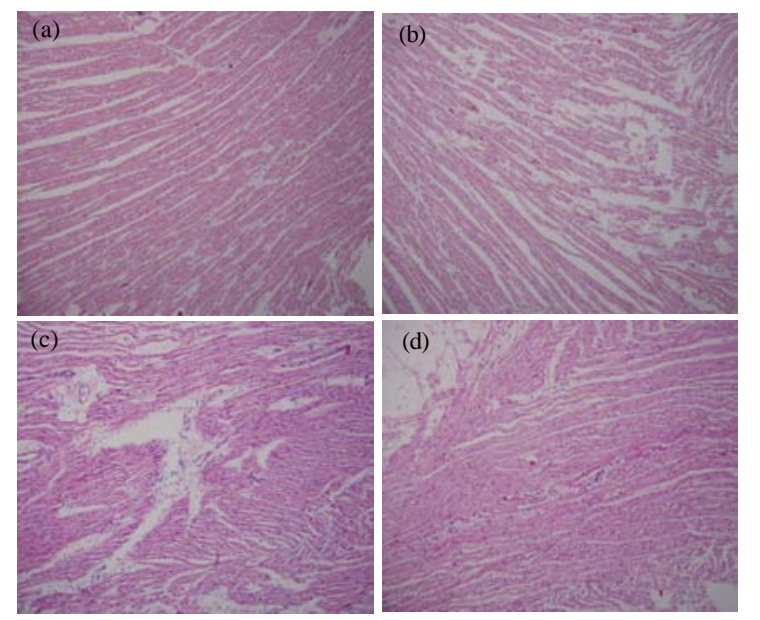

Fig. 5: Histological morphology of myocardium stained with $H \& E$ (magnification $x 100$ ); a) section of Sham group and $b$ ) the section of control group showed myocardial cell loss; c) and d) the sections of animals in groups that transplanted single and multiple doses of SHEDs, respectively; more myocardial cells and neovascularization surrounding cardiomyocyte area. Sections from SHEDs-transplanted groups

Researchers have demonstrated that intravenous injection of single and multiple dose of $1 \times 10^{6}$ SHEDs after myocardial infarction successfully promotes the cardiac repair compared with non-treated animals and improvement of cardiac autonomic modulation. Paracrine effects are possibly a mechanism that enhances ischemic tissue regeneration such as neovascularization reduced cardiac tissue inflammation and remodeling of the myocardium (Hansson et al., 2009; Perez-Ilzarbe et al., 2008). Many studies have identified the paracrine factors that possibly help to repair the cardiac tissue such as VEGF, FGF, IGF and SDF (Gnecchi et al., 2012). However, understanding the paracrine mechanism of SHEDs for regenerative therapy requires further studies to support the finding. Furthermore, although, SHED-transplanted cells express cardiac muscle cell phenotype there is no obvious evidence for electromechanical cell coupling. A long term following up in heart is needed to study the mechanism of cells therapeutic actions. On the other hand, recent study showed that transplantation of grafted cells is attributed to the lack of host-graft ectromechanical integration (Huang et al., 2011). This results in heterogeneity of conduction and delay which can eventually lead to ventricular arrhythmias (Halbach et al., 2007; Roell et al., 2007). As can be observed in the study, heart rate of animals treated with
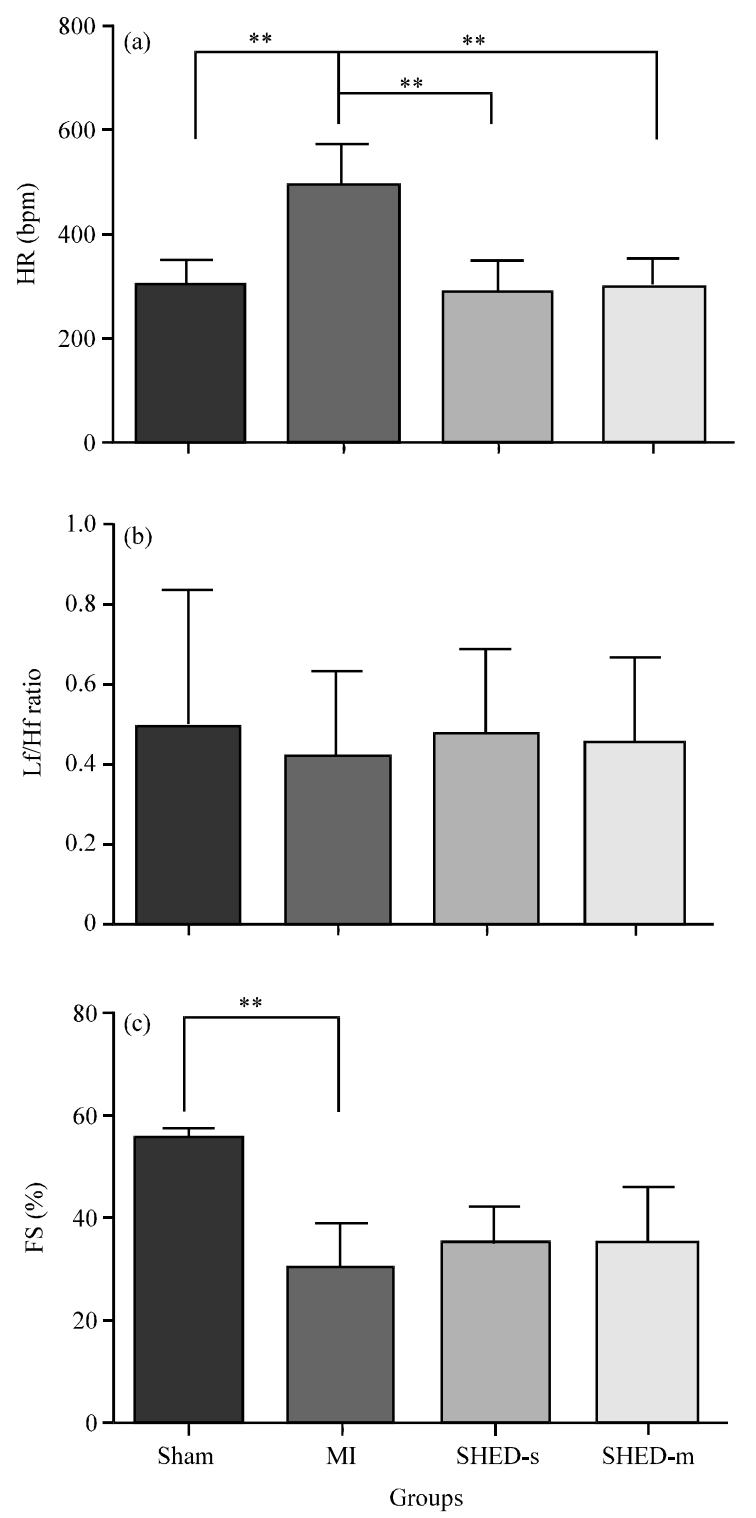

Fig. 6: a) Correlations among heart rate and b) indices of the power specttral analysis of heart rate variability and c) Fraction Shortening (FS); ${ }^{* *} \mathrm{p}<0.01$

SHEDs markedly decreases when compared with that of untreated ones. Although, no significant reduction in heart rate variability has been found, there is a positive tendency towards reduction in the cardiovascular sympathetic activity. The sympathetic nervous system appeared to play a compensatory role in the circulatory modification of heart failure (Kishi, 2012). Sympathetic nerve served as a link between the brain and heart muscle and had a significant effect on myocardial function (Kishi and Hirooka, 2012). Previous studies showed that 
autonomic nervous balance is decreases and sympathetic nervous activity is increases in chronic heart failure associated with MI (Nolan et al., 1992). In this study, researchers showed that SHEDs transplanted group improved the heart rate reflecting the reduction in sympathetic nervous activity. The findings seems to support that SHEDs therapy provided a benefit for myocardial repair however ascertaining this still required a further study of electrophysiology consideration.

\section{CONCLUSION}

Researchers study the therapeutic effects of Stem cells from Human Exfoliated Deciduous Teeth (SHED) in rabbit model. Chronic Myocardial Infarction (MI) in rabbits is induced by ligation of the left circumflex coronary artery. Researchers aim to investigate the regeneration of infarcted myocardium after SHEDs transplantation. Compared with the MI-control group infarcted size in SHEDs transplanted M group significantly decreases. Multiple doses of SHED transplantation after chronic myocardial infarction tended to give more efficiently than single injection. The most important finding of this study is that dental tissue derived stem cells administered to ischemic myocardium through an intravenous route exert therapeutic benefit. SHEDs therapy with a simple approach could provide an alternative approach for cardiac repair without ethical concerns.

\section{ACKNOWLEDGEMENTS}

This study was supported by National Science and Technology Development Agency (NSTDA) grants SCH-NR2010-11-05.

\section{REFERENCES}

Brehm, M., T. Zeus and B.E. Strauer, 2002. Stem cells: Clinical application and perspectives. Herz, 27: 611-620.

Caspi, O. and L. Gepstein, 2006. Regenerating the heart using human embryonic stem cells: From cell to bedside. Israel Med. Assoc. J., 8: 208-214.

Da Silva Meirelles, L. and N.B. Nardi, 2003. Murine marrow-derived mesenchymal stem cell: Isolation, in vitro expansion and characterization. $\mathrm{Br}$. $\mathrm{J}$. Haematol., 123: 702-711.
Fuchs, S., R. Kornowski, G. Weisz, L.F. Satler and P.C. Smits et al., 2006. Safety and feasibility of transendocardial autologous bone marrow cell transplantation in patients with advanced heart disease. Am. J. Cardiol., 97: 823-829.

Gandia, C., A. Arminan, J.M. Garcia-Verdugo, E. Lledo and A. Ruiz et al., 2008. Human dental pulp stem cells improve left ventricular function, induce angiogenesis and reduce infarct size in rats with acute myocardial infarction. Stem Cells, 26: 638-645.

Gepstein, L., 2002. Derivation and potential applications of human embryonic stem cells. Circ. Res., 91: 866-876.

Gnecchi, M., P. Danieli andE. Cervio, 2012. Mesenchymal stem cell therapy for heart disease. Vasc. Pharmacol., 57: 48-55.

Halbach, M., K. Pfannkuche, F. Pillekamp, A. Ziomka and T. Hannes et al., 2007. Electrophysiological maturation and integration of murine fetal cardiomyocytes after transplantation. Circ. Res., 101: 484-492.

Hansson, E.M., M.E. Lindsay and K.R. Chien, 2009. Regeneration next: Toward heart stem cell therapeutics. Cell Stem Cell, 5: 364-377.

Henning, R.J., J.D. Burgos, M. Vasko, F. Alvarado, C.D. Sanberg, P.R. Sanberg and M.B. Morgan, 2007. Human cord blood cells and myocardial infarction: Effect of dose and route of administration on infarct size. Cell Transplantation, 16: 907-917.

Huang, G., M. Pashmforoush, B. Chung and L.A. Saxon, 2011. The role of cardiac electrophysiology in myocardial regenerative stem cell therapy. J. Cardiovasc. Translational Res., 4: 61-65.

Huang, G.T., S. Gronthos and S. Shi, 2009. Mesenchymal stem cells derived from dental tissues vs. those from other sources: Their biology and role in regenerative medicine. J. Dental Res., 88: 792-806.

Kishi, T. and Y. Hirooka, 2012. Central mechanisms of abnormal sympathoexcitation in chronic heart failure. Cardiol. Res. Pract. 10.1155/2012/847172.

Kishi, T., 2012. Heart failure as an autonomic nervous system dysfunction. J. Cardiol., 59: 117-122.

Nolan, J., A.D. Flapan, S. Capewell, T.M. MacDonald, J.M. Neilson and D.J. Ewing, 1992. Decreased cardiac parasympathetic activity in chronic heart failure and its relation to left ventricular function. Br. Heart J., 67: 482-485.

Perez-Ilzarbe, M., O. Agbulut, B. Pelacho, C. Ciorba and E. San Jose-Eneriz et al., 2008. Characterization of the paracrine effects of human skeletal myoblasts transplanted in infarcted myocardium. Eur. J. Heart Failure, 10: 1065-1072. 
Petersen, T. and L. Niklason, 2007. Cellular lifespan and regenerative medicine. Biomaterials, 28: 3751-3756.

Roell, W., T. Lewalter, P. Sasse, Y.N. Tallini and B.R. Choi et al., 2007. Engraftment of connexin 43-expressing cells prevents post-infarct arrhythmia. Nature, 450: 819-824.
Roger, V.L., A. S. Go, D.M. Lloyd-Jones, E.J. Benjamin and J.D. Berry et al., 2012. Heart disease and stroke statistics-2012 update: A report from the American Heart Association. Circulation, 125: e2-e220.

Tomita, S., R.K. Li, R.D. Weisel, D.A.G. Mickle, E.J. Kim, T. Sakai and Z.Q. Jia, 1999. Autologous transplantation of bone marrow cells improves damaged heart function. Circulation, 100: II247-II256. 